\title{
SOLUBILITY OF DEUTERIUM IN ALUMINA NANOPOWDERS
}

\author{
V. B. Vykhodets, T. E. Kurennykh \\ M.N. Miheev Institute of Metal Physics of Ural Branch of Russian Academy of Sciences, 18 S. Kovalevskoy st., \\ Ekaterinburg, Russian Federation
}

\begin{abstract}
*Corresponding author. E-mail: kurennykh@imp.uran.ru, address for correspondence: 18, ul. S. Kovalevskoy, 620990, Ekaterinburg, Russian Federation. Tel.: +7 34337838 46; fax: +7 3433745244
\end{abstract}

The paper presents data on the effect of surface oxygen deficiency in nanoparticles on the catalytic properties of oxide nanopowders. Data on the interaction of nanopowders with gaseous hydrogen are the test modeling catalytic properties. Alumina nanopowders synthesized using laser evaporation of a ceramic target dissolve deuterium during their annealing in gaseous deuterium. It is only the surface atomic layer of nanoparticles with very large oxygen deficiency that has the ability to dissolve deuterium. Oxygen deficiency and deuterium solubility are adjustable properties of nanoparticles. They can be changed by annealing nanopowders in oxygen. Stoichiometric alumina virtually does not dissolve deuterium. A technological scheme is proposed for synthesizing oxide nanopowders with controllable surface oxygen deficiency, physical-chemical and functional properties.

Keywords: deuterium, alumina nanopowder, catalytic properties, oxygen nonstoichiometry, nuclear reaction analysis.

DOI: $10.17804 / 2410-9908.2016 .5 .006-014$

\section{References}

1. Vykhodets V.B., Jarvis E., Kurennykh T.E., Davletshin A.E., Obukhov S.I., Beketov I.V., Samatov O.M., Medvedev A.I. Extreme deviations from stoichiometry in alumina nanopowders. Surface Science, 2014, vol. 630, pp. 182-186. DOI: 10.1016/j.susc.2014.08.009.

2. Vykhodets V.B., Jarvis E., Kurennykh T.E., Beketov I.V., Obukhov S.I., Samatov O.M., Medvedev A.I., Davletshin A.E., Whyte T. Inhomogeneous depletion of oxygen ions in oxide nanoparticles. Surface Science, 2016, vol. 644, pp. 41-147. DOI: 10.1016/j.susc.2015.10.011.

3. Jarvis E.A.A., Carter E.A. Metallic Character of the $\mathrm{Al}_{2} \mathrm{O}_{3}(0001)-(\sqrt{31} \times \sqrt{31}) \mathrm{R} \pm 9^{\circ}$ Surface Reconstruction. J. Phys. Chem B, 2001, vol. 105, iss. 18, pp. 4045-4052. DOI: $10.1021 / j p 003587 \mathrm{c}$.

4. French T.M., Somorjai Gabor A. Composition and surface structure of the (0001) face of $\alpha$ alumina by low-energy electron diffraction. J. Phys. Chem., 1970, vol. 74, no. 12, pp. 2489-2495.

5. Sundaresan A., Bhargavi R., Rangarajan N., Siddesh U., Rao C. N. R. Ferromagnetism as a universal feature of nanoparticles of the otherwise nonmagnetic oxides. Phys. Rev. B, 2006, vol. 74, iss. 16, pp. 161306(R). DOI: 10.1103/PhysRevB.74.161306.

6. $\quad$ Sudakar C., Kharel P., Suryanarayanan R., Thakurc J.S., Naikd V.M., Naika R., Lawes G. Room temperature ferromagnetism in vacuum-annealed $\mathrm{TiO}_{2}$ thin films. JMMM, 2008, vol. 320, iss. 5, pp. L31-L36. DOI: 10.1016/j.jmmm.2007.07.026.

7. Hong N., Sakai J., Poirot N., Brisé V. Room-temperature ferromagnetism observed in undoped semiconducting and insulating oxide thin films. Phys. Rev. B, 2006, vol. 73, iss. 13, pp. 132404. DOI: 10.1103/PhysRevB.73.132404.

8. Coey J.M.D. High-temperature ferromagnetism in dilute magnetic oxides. J. Appl. Phys., 2005, vol. 97, iss. 10, part 2, pp. 10D313. DOI: 10.1063/1.1849054.

9. Venkatesan M., Fitzgerald C.B., Coey J.M.D. Unexpected magnetism in a dielectric oxide. Nature, 2004, vol. 430, iss. 7000, pp. 630. DOI: 10.1038/430630a. 
10. Dutta P., Seehra M.S., Zhang Y., Wender I. Nature of magnetism in copper-doped oxides: $\mathrm{ZrO}_{2}, \mathrm{TiO}_{2}, \mathrm{MgO}, \mathrm{SiO}_{2}, \mathrm{Al}_{2} \mathrm{O}_{3}$, and ZnO. J. Appl. Phys., 2008, vol. 103, iss. 7, pp. 07D104. DOI: $10.1063 / 1.2830555 /$.

11. Coey J.M.D., Venkatesan M., Stamenov P., Fitzgerald C.B., Dorneles L.S. Magnetism in hafnium dioxide. Phys. Rev. B, 2005, vol. 72, iss. 2, pp. 024450. DOI: 10.1103/PhysRevB.72.024450.

12. Vykhodets V.B., Klotsman S.M, Levin A.D. Oxygen diffusion in alpha Titanium. 2. Calculation of concentrational profile of impurity by nuclear microanalysis. Fizika Metallov $i$ Metallovedenie, 1987, vol.64, iss. 5, pp. 920-923. 
Подана в журнал: 24.10.2016

УДК 544.6:544.16:539.2:517.958

DOI: $10.17804 / 2410-9908.2016 .5 .006-014$

\title{
РАСТВОРИМОСТЬ ДЕЙТЕРИЯ В НАНОПОРОШКАХ ОКСИДА АЛЮМИНИЯ
}

\author{
В. Б. Выходец, Т. Е. Куренных*
}

\begin{abstract}
Федеральное государственное бюджетное учреждение науки Институт физики металлов имени М.Н. Михеева Уральского отделения Российской академии наук, ул. С. Ковалевской, 18, Екатеринбург, Российская Федерация

*Ответственный автор. Электронная почта: kurennykh@imp.uran.ru, адрес для переписки: 620990, ул. С. Ковалевской, 18, Екатеринбург, Россия. Телефон: +7 343 378-38-46; факс: +7 343 374-52-44
\end{abstract}

В работе получены данные о влиянии поверхностного кислородного дефицита в наночастицах на каталитические свойства оксидных нанопорошков. Тестом, моделирующим каталитические свойства, были данные о взаимодействии нанопорошков с газообразным водородом. Нанопорошки оксида алюминия, синтезированные с помощью технологии лазерного испарения керамической мишени, растворяют дейтерий при их отжиге в газообразном дейтерии. Способностью растворять дейтерий обладает только поверхностный атомный слой наночастиц, в котором имеет место дефицит кислорода в сравнении со стехиометрией. Дефицит кислорода и растворимость дейтерия являются регулируемыми свойствами наночастиц. Они могут быть изменены при отжиге нанопорошков в кислороде. Оксид алюминия стехиометрического состава практически не растворяет дейтерий. Предложена технологическая схема, в рамках которой могут быть синтезированы оксидные нанопорошки с регулируемым поверхностным кислородным дефицитом, физико-химическими и функциональными свойствами.

Ключевые слова: дейтерий, нанопорочок, каталитические свойства, кислородная нестехиометрия, ядерный микроанализ, оксид алюминия.

\section{1. Введение}

Оксидные нанопорошки являются важными функциональными материалами, они широко применяются в машиностроении, катализе, медицине, химических и электрохимических устройствах, атомной, ядерной и водородной энергетике. В настоящее время мировое производство оксидных нанопорошков превышает 100 тыс. тонн. В связи с этим, актуальными задачами являются исследование свойств оксидных нанопорошков и разработка стратегии их применения в технике. Традиционно наиболее важными свойствами порошков являются их химический и фазовый состав, а также средний размер наночастиц. Именно эти характеристики в основном учитываются в стратегии применения нанопорошков. В то же время в последнее время появились данные, свидетельствующие о том, что концентрация кислорода в приповерхностной области кристаллической решетки оксидов ниже, чем в регулярной решетке $[1,2]$. По результатам этих работ толщина дефицитного по кислороду слоя незначительна, она сопоставима с размером атома. Такого типа информация представляет большой интерес для физики и химии поверхности и для многих приложений, связанных с электронными, магнитными, каталитическими и другими свойствами оксидов. Особенно она актуальна для оксидных наночастиц, поскольку на их поверхности расположена большая доля атомов системы. Отклонение от стехиометрии в поверхностном атомном слое оксидных наночастиц может оказывать сильное влияние на свойства оксидных нанопорошков и, как следствие, на стратегию их применения. Однако в настоящее время в литературе отсутствуют экспериментальные данные, свидетельствующие о чувствительности каких-либо физикохимических и функциональных свойств оксидных нанопорошков к поверхностному кисло- 
родному дефициту, а также сведения об использовании этого эффекта в практических приложениях.

Имеющаяся в литературе информация о структуре и свойствах дефицитного по кислороду поверхностного слоя в оксидах, в том числе в наночастицах, очень ограниченна [311]. Объективно получение таких данных с помощью многих традиционных методик, например рентгеновской фотоэлектронной спектроскопии, осложнено из-за очень малой толщины дефицитного по кислороду слоя. Самостоятельный интерес представляют также данные о влиянии поверхностного кислородного дефицита на функциональные свойства нанопорошков. В связи с этим основной задачей настоящей работы было получение данных о влиянии поверхностного кислородного дефицита в наночастицах на каталитические свойства оксидных нанопорошков. Тестом, моделирующим каталитические свойства, были данные о взаимодействии нанопорошков с газообразным водородом. Предметом исследования была растворимость дейтерия в нанопорошках оксида алюминия. Выбор объекта исследований определялся тем, что нанопорошки оксида алюминия широко применяются в технике, и в литературе имеются данные о поверхностном кислородном дефиците в наночастицах этого материала $[1,3]$. Использование дейтерия было обусловлено тем, что он является малораспространенным изотопом водорода, поэтому легко было исключить неконтролируемое поступление в образцы атомов водорода из окружающей атмосферы.

\section{2. Образцы и методики}

Исследование проведено на нанопорошках диоксида алюминия, синтезированных с помощью технологии лазерного испарения керамической мишени. Подробное описание методик синтеза и результатов исследования нанопорошков представлено в работе [1]. Здесь приведем только данные, которые принимались во внимание при выполнении данной работы. Кислородная подсистема нанопорошков была обогащена малораспространенным изотопом ${ }^{18} \mathrm{O}$, т.е. порошки имели состав $\mathrm{Al}^{16} \mathrm{O}_{\mathrm{x}}{ }^{18} \mathrm{O}_{\mathrm{y}}$. С помощью методики ядерного микроанализа были проведены измерение средних концентраций изотопов ${ }^{16} \mathrm{O}$ и ${ }^{18} \mathrm{O}$ в нанопорошках. Эти исследования показали, что значения индекса $y$, характеризующего содержание изотопа ${ }^{18} \mathrm{O}$ в кристаллической решетке наночастиц, при возрастании удельной поверхности $S$ в диапазоне от 55 до $267 \mathrm{~m}^{2} / \Gamma$ сильно уменьшались, почти в 2 раза. Соответствующая зависимость $y(S)$ была линейной.

Такие данные свидетельствовали о наличии кислородного дефицита в оксидных наночастицах к моменту завершения высокотемпературного синтеза и о локализации дефицита в тонком наружном слое наночастиц. Его толщина была оценена из данных по $y(S)$ и составила 0,37 нм. Кислородный индекс $x(S)$, характеризующий содержание изотопа ${ }^{16} \mathrm{O}$ в порошках, в отличие от $y(S)$ практически не зависел от $S$. Это означало, что после завершения высокотемпературного синтеза, т.е. при остывании и хранении порошков протекали процессы, во время которых атомы ${ }^{16} \mathrm{O}$ поступали в нанопорошки из окружающей атмосферы. В общем случае это могли быть процессы окисления тонкого поверхностного слоя наночастиц, а также хемосорбция и адсорбция наночастицами кислородосодержащих молекул.

Таким образом, в работе [1] были зарегистрированы два отличающихся друг от друга состояния наночастиц оксида алюминия. Первое из них является термодинамически равновесным при высокой температуре и реализуется к моменту завершения высокотемпературного синтеза. В этом состоянии имеет место очень большой дефицит кислорода в поверхностном атомном слое кристаллической решетки наночастиц и не исключено, что атомы кислорода вообще отсутствуют в поверхностном слое. Второе состояние является низкотемпературным, оно сформировалось в процессе остывания наночастиц после высокотемпературного синтеза и их взаимодействия с окружающей атмосферой. Кислородный дефицит в кристаллической решетке наночастиц в этом состоянии неизвестен, и в литературе отсутствуют методики для его измерения. Это связано с отсутствием данных о чувствительности физико- 
химических свойств нанопорошков к кислородному дефициту. Поскольку при остывании наночастиц происходило поступление атомов ${ }^{16} \mathrm{O}$ в нанопорошки, можно предполагать, что в поверхностном слое наночастиц при комнатной температуре присутствуют кислородные вакансии или другие точечные дефекты и что кислородный дефицит в низкотемпературном состоянии меньше, чем в высокотемпературном.

Высокотемпературное состояние вряд ли представляет интерес для практических приложений, и предметом исследований в настоящей работе было взаимодействие с дейтерием порошков в низкотемпературном состоянии.

Отжиги порошков в дейтерии и кислороде, обогащенном на $80 \%$ изотопом ${ }^{18} \mathrm{O}$, проводили при $400{ }^{\circ} \mathrm{C}$. Существенно понизить температуру отжигов не представлялось возможным, поскольку при более низких температурах, например при $200{ }^{\circ} \mathrm{C}$, порошки практически не взаимодействовали с газами. Это связано, вероятно, с тем, что при температурах ниже $400{ }^{\circ} \mathrm{C}$ скорость поверхностных реакций, например диссоциации молекул $\mathrm{D}_{2}$ и $\mathrm{O}_{2}$, была низкой и лимитировала скорость поступления атомов $\mathrm{D}$ и $\mathrm{O}$ в наночастицы. Отжиги проводили в кварцевой трубе. Она размещалась внутри массивного металлического цилиндра. В этих условиях градиент температуры в зоне расположения образцов практически отсутствовал. Температуру измеряли с помощью хромель-алюмелевой термопары с точностью $1{ }^{\circ} \mathrm{C}$. Продолжительность отжигов была 1 ч. Время, необходимое для достижения температуры отжига, составляло 10 мин, а время охлаждения образцов до комнатной температуры 1 мин. Отжиги в дейтерии проводили при давлении 0,6 атм, а в кислороде - при 0,3 атм. Значительных изменений фазового состава порошков при отжигах не происходило. Удельная поверхность порошков при отжигах уменьшалась не очень сильно, и это обстоятельство при обсуждении результатов во внимание не принималось. Максимальное изменение удельной поверхности составляло 1,5 раза.

Средние концентрации изотопа ${ }^{2} \mathrm{H}$ в порошках определялись с помощью методики ядерного микроанализа на 2 MV ускорителе Ван де Граафа. Для проведения измерений частицы нанопорошков оксида впрессовывались в индиевую пластину. Измерения с помощью резерфордовского обратного рассеяния показали, что в зоне анализа, глубина которой была около 1 мкм, атомы индия отсутствовали. Для измерения концентрации изотопа ${ }^{2} \mathrm{H}$ использовалась реакция ${ }^{2} \mathrm{H}(\mathrm{d}, \mathrm{p})^{3} \mathrm{H}$ при энергии дейтронов 650 кэВ. Диаметр первичного пучка дейтронов составлял 1 мм. В наших экспериментах на ускорителе в зоне анализа находилось не менее 1012 частиц нанопорошка, это обеспечивало корректное измерение средних для ансамбля наночастиц концентраций дейтерия. Регистрация продуктов ядерных реакций проводилась с помощью кремниевого поверхностно-барьерного детектора диаметром около 10 мм, угол регистрации был $160^{\circ}$. Доза облучения, т.е. число частиц первичных пучков, падающих на образец, определялась с помощью вторичного монитора, статистическая погрешность в этих измерениях была около $0,5 \%$.

При математической обработке спектров продуктов ядерных реакций использовалась приближенная процедура [12] сравнения спектров от исследуемых образцов и эталонного образца с постоянной по глубине концентрацией дейтерия. Систематическая ошибка в измерении концентраций, связанная с использованием приближенной процедуры, не превышала $1 \%$. Образцом-эталоном при измерении концентрации дейтерия был дейтерид $\mathrm{ZrCr} 2 \mathrm{D}_{4}$. Среднеквадратичные ошибки при измерениях концентрации изотопа ${ }^{2} \mathrm{H}$ составляли от 2 до $6 \%$.

\section{3. Результаты и обсуждение}

При исследовании взаимодействия нанопорошков с дейтерием было установлено, что микропорошок оксида алюминия, имеющий удельную поверхность около $2 \mathrm{~m}^{2} / \Gamma$, практически не растворял дейтерий. Литературные данные по растворимости водорода и дейтерия в оксиде алюминия отсутствуют. После отжигов в дейтерии мы регистрировали очень низкие 
концентрации дейтерия в микропорошке $\mathrm{Al}_{2} \mathrm{O}_{3}$ - менее 0,1 ат. \%. Присутствие дейтерия в небольших количествах в микропорошке было обусловлено имплантацией ионов дейтерия в образцы при использовании реакции ${ }^{2} \mathrm{H}(\mathrm{d}, \mathrm{p}){ }^{3} \mathrm{H}$ для измерения концентрации дейтерия с помощью методики ядерного микроанализа.

В то же время нанопорошки поглощали значительные количества дейтерия при их отжиге в газообразном дейтерии, соответствующие данные приведены на рис. 1 и 2 . Из них видно, что концентрация дейтерия $C d$ возрастала при увеличении удельной поверхности порошков $S$. Об этом свидетельствовали зависимости $C d(S)$ на рис. 2, а также спектры продуктов ядерных реакций, представленные на рис. 1. Параметром, зависящим от концентрации дейтерия в нанопорошках, является высота спектра реакции ${ }^{2} \mathrm{H}(\mathrm{d}, \mathrm{p})^{3} \mathrm{H}$, на рис. 1 она возрастала при увеличении $S$. На основании полученных результатов по взаимодействию микро- и нанопорошков оксида алюминия с газообразным дейтерием можно заключить, что способностью поглощать дейтерий из газовой фазы обладает только дефицитный по кислороду поверхностный слой наночастиц.

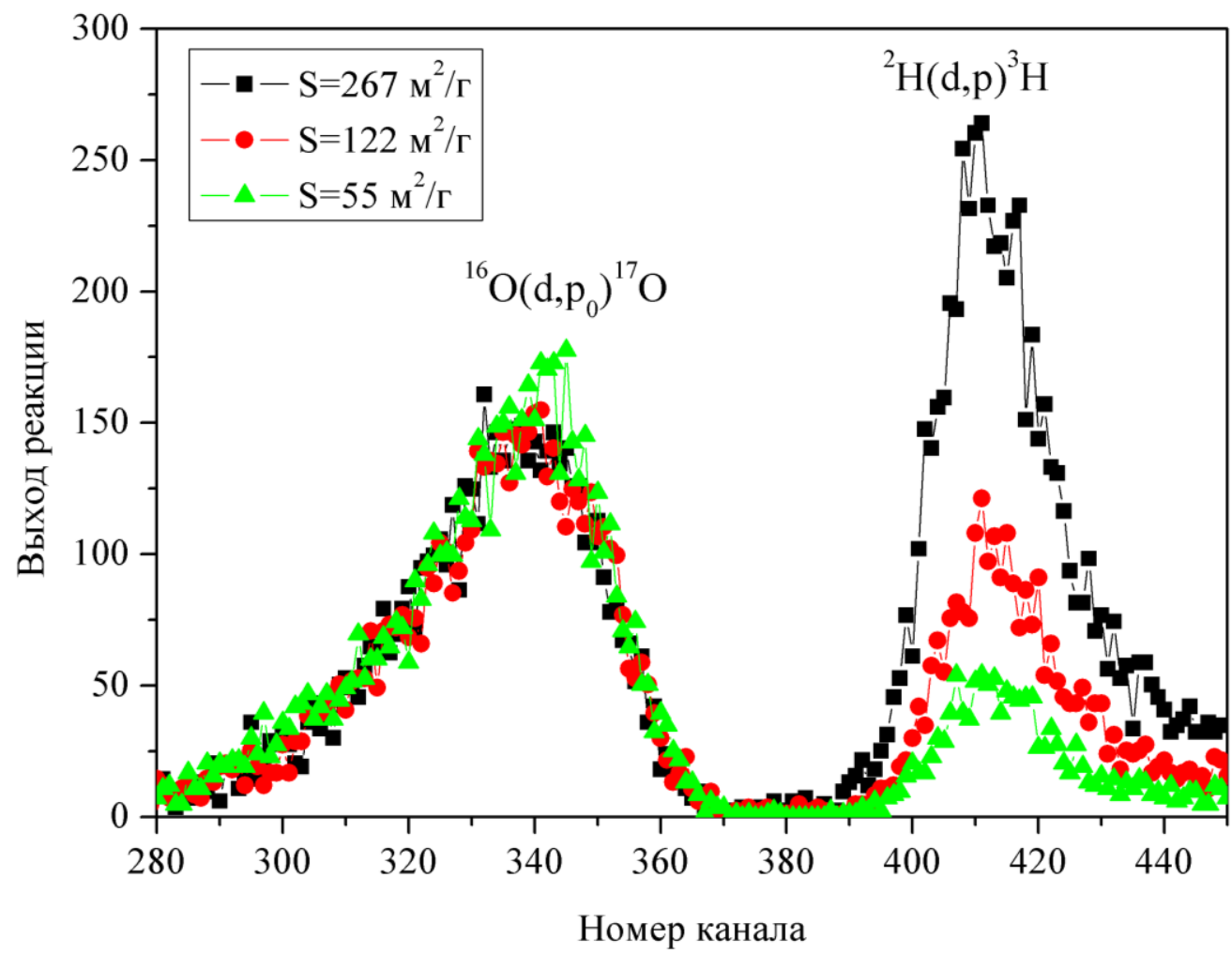

Рис. 1. Спектры продуктов ядерных реакций, полученные при облучении

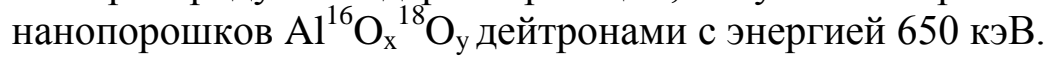

Дозы облучения всех порошков были одинаковыми

Отметим также две особенности спектров, приведенных на рис. 1. Форма спектров для реакции ${ }^{2} \mathrm{H}(\mathrm{d}, \mathrm{p})^{3} \mathrm{H}$ соответствовала образцам с постоянной по глубине концентрацией дейтерия. Этот результат не противоречит заключению о локализации атомов дейтерия в поверхностном слое наночастиц, поскольку толщина этого слоя была много меньше линейного размера наночастиц и разрешения методики ядерного микроанализа по глубине в образце. В нашем случае [1] толщина дефицитного по кислороду слоя составляет примерно 0,4 нм, размер наночастиц был в интервале от 3 до 15 нм, а разрешение методики по глубине - не лучше 50 нм. Кроме того, спектры реакции ${ }^{16} \mathrm{O}\left(\mathrm{d}, \mathrm{p}_{1}\right)^{17} \mathrm{O}$ на рис. 1 практически совпадали для порошков с различной удельной поверхностью, что связано с примерно одинаковой концентрацией изотопа ${ }^{16} \mathrm{O}$ во всех нанопорошках. 
Таким образом, в работе впервые были получены данные о влиянии поверхностного кислородного дефицита в оксидных наночастицах на одно из важных функциональных свойств нанопорошков. Таким свойством является растворимость дейтерия в нанопорошках, которую можно рассматривать как параметр, характеризующий каталитические свойства материала. Этот результат представляет интерес не только для катализа. В разд. 2 отмечалось, что из-за отсутствия данных о чувствительности каких-либо физико-химических свойств нанопорошков к кислородному дефициту до настоящего времени не были разработаны методики и подходы для измерения поверхностного кислородного дефицита в кристаллической решетке оксидных наночастиц. Результаты этой работы показывают, что эта задача может быть решена посредством измерения растворимости дейтерия в нанопорошках. Этот вопрос нуждается в дополнительных исследованиях. В них, в частности, должны быть получены экспериментальные и теоретические данные о взаимодействии с дейтерием кислородных вакансий и других точечных дефектов, ответственных за поверхностный кислородный дефицит в оксидных наночастицах.

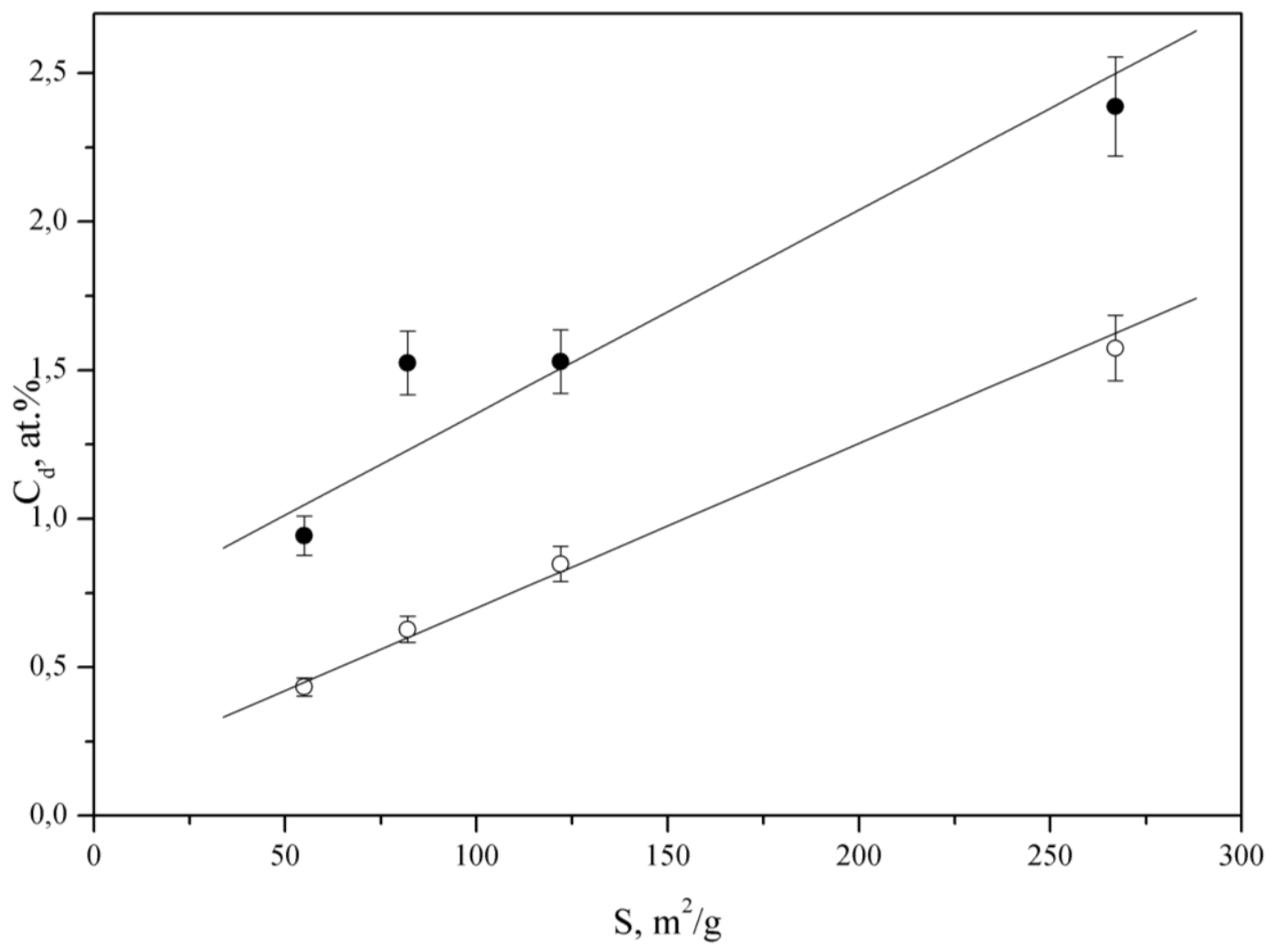

Рис.2. Зависимость средней концентрации дейтерия $\mathrm{C}_{\mathrm{d}}$ от удельной поверхности $S$ в нанопорошках оксида алюминия: • - после отжига в $\mathrm{D}_{2}$ при $400{ }^{\circ} \mathrm{C}$ и $\mathrm{p}=0,6$ атм; о - после отжига в $\mathrm{D}_{2}$ при $400{ }^{\circ} \mathrm{C}$ и $\mathrm{p}=0,6$ атм и последующего отжига в ${ }^{18} \mathrm{O}$ при $400{ }^{\circ} \mathrm{C}$ и $\mathrm{p}=0,3$ атм

Как отмечалось во введении, в настоящее время эффект поверхностного кислородного дефицита в оксидных наночастицах практически не принимается во внимание в стратегии применения нанопорошков. Результаты этой работы позволяют сформировать подход к синтезу и обработке оксидных нанопорошков, в рамках которого эффект поверхностного кислородного дефицита будет использован для получения оксидных порошков с регулируемыми свойствами. Он основан на следующих соображениях. В наших работах $[1,2]$ было показано, что при высокотемпературном синтезе нанопорошков с помощью технологии лазерного испарения керамической мишени формируются наночастицы с очень большим дефицитом кислорода в поверхностном атомном слое. При остывании и хранении нанопорошков происходит взаимодействие наночастиц с окружающей атмосферой. При этом взаимодействии кис- 
лородный дефицит уменьшается, изменяются состав и структура поверхностного слоя и функциональные свойства нанопорошков. По данным этой работы могут быть получены оксидные нанопорошки, которые растворяют дейтерий, причем растворимость дейтерия является регулируемым свойством, что видно из рис. 2, где представлены данные о растворимости дейтерия в нанопорошках оксида алюминия до и после отжига образцов в кислороде. Этот результат показывает, что в принципе может быть получен широкий спектр структур поверхностного слоя наночастиц, если обеспечить контролируемые и регулируемые условия охлаждения порошков после синтеза, а также проводить отжиги порошков в кислороде после завершения синтеза. Это направление исследований, по нашему мнению, является перспективным для синтеза оксидных нанопорошков с различными электрическими, магнитными, каталитическими и другими функциональными свойствами.

\section{4. Заключение}

- $\quad$ Нанопорошки оксида алюминия, синтезированные с помощью технологии лазерного испарения керамической мишени, растворяют дейтерий при их отжиге в газообразном дейтерии.

- $\quad$ Способностью растворять дейтерий обладает только поверхностный атомный слой наночастиц, в котором имеет место дефицит кислорода в сравнении со стехиометрией. Дефицит кислорода и растворимость дейтерия являются регулируемыми свойствами наночастиц. Они могут быть изменены при отжиге нанопорошков в кислороде. Оксид алюминия стехиометрического состава практически не растворяет дейтерий.

- $\quad$ Предложена технологическая схема, в рамках которой могут быть синтезированы оксидные нанопорошки с регулируемым поверхностным кислородным дефицитом, физикохимическими и функциональными свойствами.

\section{Благодарность}

Работа выполнена в рамках проекта УрО РАН №15-17-2-10 при частичной поддержке РФФИ (проект №15-03-06617).

\section{Литература}

1. Extreme deviations from stoichiometry in alumina nanopowders / V. B. Vykhodets, E. Jarvis, T. E. Kurennykh, A. E. Davletshin, S. I. Obukhov, I. V. Beketov, O. M. Samatov, A. I. Medvedev // Surface Science. - 2014. - Vol. 630. - P. 182-186. DOI: 10.1016/j.susc.2014.08.009.

2. Inhomogeneous depletion of oxygen ions in oxide nanoparticles / V. B. Vykhodets, E. Jarvis, T. E. Kurennykh, I. V. Beketov, S. I. Obukhov, O. M. Samatov, A. I. Medvedev, A. E. Davletshin, T. Whyte // Surface Science. - 2016. - Vol. 644. - P. 141-147. DOI: 10.1016/j.susc.2015.10.011.

3. Jarvis E. A. A., Carter E. A. Metallic Character of the $\mathrm{Al}_{2} \mathrm{O}_{3}(0001)-(\sqrt{31} \times \sqrt{31}) \mathrm{R} \pm 9^{\circ}$ Surface Reconstruction // J. Phys. Chem. B. - 2001. - Vol. 105, iss. 18. - P. 4045-4052. DOI: $10.1021 / \mathrm{jp} 003587 \mathrm{c}$.

4. French T. M, Somorjai Gabor A. Composition and surface structure of the (0001) face of $\alpha$-alumina by low-energy electron diffraction // J. Phys. Chem. - 1970. - Vol. 74, no. 12. P. 2489-2495.

5. Ferromagnetism as a universal feature of nanoparticles of the otherwise nonmagnetic oxides / A. Sundaresan, R. Bhargavi, N. Rangarajan, U. Siddesh, C. N. R. Rao // Phys. Rev. B. - 2006. Vol. 74, iss. 16. - P. 161306(R). - DOI: 10.1103/PhysRevB.74.161306. 
6. Room temperature ferromagnetism in vacuum-annealed $\mathrm{TiO}_{2}$ thin films / C. Sudakar, P. Kharel, R. Suryanarayanan, J. S. Thakurc, V. M. Naikd, R. Naika, G. Lawes // JMMM. - 2008. Vol. 320, iss. 5. - DOI: 10.1016/j.jmmm.2007.07.026.

7. Room-temperature ferromagnetism observed in undoped semiconducting and insulating oxide thin films / N. Hong, J. Sakai, N. Poirot, V. Brisé // Phys. Rev. B. - 2006. - Vol. 73, iss. 13. P. 132404. - DOI: 10.1103/PhysRevB.73.132404.

8. Coey J. M. D. High-temperature ferromagnetism in dilute magnetic oxides // J. Appl. Phys. - 2005. - Vol. 97, iss. 10, part 2. - P. 10D313. - DOI: 10.1063/1.1849054.

9. Venkatesan M., Fitzgerald C. B., Coey J. M. D. Unexpected magnetism in a dielectric oxide // Nature. - 2004. - Vol. 430, iss. 7000. - P. 630. - DOI: 10.1038/430630a.

10. Nature of magnetism in copper-doped oxides: $\mathrm{ZrO}_{2}, \mathrm{TiO}_{2}, \mathrm{MgO}, \mathrm{SiO}_{2}, \mathrm{Al}_{2} \mathrm{O}_{3}$, and $\mathrm{ZnO} /$ P. Dutta, M. S. Seehra, Y. Zhang, I. Wender // J. Appl. Phys. - 2008. - Vol.103, iss. 7. P. 07D104. - DOI: 10.1063/1.2830555/.

11. Magnetism in hafnium dioxide / J. M. D. Coey, M. Venkatesan, P. Stamenov, C. B. Fitzgerald, L. S. Dorneles // Phys. Rev. B. - 2005. - Vol. 72. - P. 024450. DOI: 10.1103/PhysRevB.72.024450.

12. Vykhodets V. B., Klotsman S. M, Levin A. D. Oxygen diffusion in $\alpha$-Ti. II. The calculation of the concentration profile of impurities in the nuclear microanalysis // Fizika Metallov $i$ Metallovedenie. - 1987. - Vol.64, iss. 5. - P. 920-923. 